\title{
A SOLUTION OF A PROBLEM OF OPPEHEIM
}

\author{
LING ZHU
}

Abstract. In this paper, the open problem proposed by Oppeheim [A. Oppeheim, E1277, The American Mathematical Monthly, 64, (1957) p. 504] is discussed carefully. At the same time, the Shafer, Fink and Malesevic inequalities are deduced from the solution of Oppeheim's problem.

Mathematics subject classification (2000): $26 \mathrm{D} 15$.

Key words and phrases: Oppeheim's problem, Shafer inequality, Fink inequality, Malesevic inequality.

\section{REFERENCES}

[1] D. S. Mitrinovic, Analytic Inequalities, Springer-Verlag, 1970.

[2] A. M. FInK, Two Inequalities, Univ. Beograd. Publ. Elektrotehn. Fak. 6 (1995), 49-50.

[3] L. ZHU, On Shafer-Fink Inequalities, Mathematical Inequalities and Applications 8 no. 4 (2005), $571-574$.

[4] B. J. MALESEVIC, Application of $\lambda$-Method on Shafer-Fink Inequality, Univ. Beograd. Publ. Elektrotehn. Fak. 8 (1997), 103-105.

[5] A. OPPEHEIM, E1277, The American Mathematical Monthly 64 (1957), 504.

[6] W. B. CARVER, Extreme Parameters in an Inequality, The American Mathematical Monthly 65 (1958), 206-209.

[7] G. D. Anderson, M. K. VAmanamurthy And M. Vuorinen, Conformal Invariants Inequalities and Quasiconformal Maps, New York, 1997.

[8] G. D. Anderson, S.-L. Qiu M. K. Vamanamurthy And M. Vuorinen, Generalized elliptic integral and modular equations, Pacific J. Math. 192 (2000), 1-37. 